\title{
Cerebral endothelial dysfunction in reversible cerebral vasoconstriction syndrome: a case-control study
}

\author{
Hyun Ah Choi ${ }^{\dagger}$, Mi Ji Lee ${ }^{\dagger}$ and Chin-Sang Chung ${ }^{*}$
}

\begin{abstract}
Background: The aim of this study is to investigate cerebral endothelial dysfunction in patients with reversible cerebral vasoconstriction syndrome (RCVS).

Methods: We prospectively recruited patients with RCVS, age-matched controls with episodic migraine, and age-matched healthy controls at Samsung Medical Center from Apr 2015 to Jul 2016. All participants underwent transcranial Doppler evaluation, with a breath-holding maneuver, for the evaluation of bilateral middle cerebral arteries (MCAs), posterior cerebral arteries (PCAs), and the basilar artery (BA). The breath-holding index (BHI) was used to measure cerebral endothelium-dependent vasodilation. Follow-up BHIs were recorded in selected patients with RCVS after 3 months.
\end{abstract}

Results: A total of 84 subjects were recruited for this study ( $n=28$ in each group of RCVS, episodic migraine, and healthy control; mean age, 49.8 years). The RCVS group showed lower BHIs in all basal arteries, in comparison to healthy controls ( $p<0.001,0.009$ for bilateral MCAs, $p<0.001$ and 0.028 for bilateral PCAs, and $p=0.060$ for the BA). Compared to migraineurs, RCVS patients had lower BHIs only in the anterior circulation ( $p=0.002$ and 0.038 for bilateral MCAs; $p=0.069$ and 0.247 for bilateral PCAs; $p=0.120$ for the BA). Of the 10 patients who had follow-up $\mathrm{BH}$ s at 3 months, 7 showed complete normalization, while three did not.

Conclusions: Cerebral endothelial function is impaired in a widespread distribution in RCVS. Its role in the pathogenesis and clinical outcome of RCVS should be determined in further studies.

Keywords: Reversible cerebral vasoconstriction syndrome, Pathophysiology, Endothelial dysfunction, Breath holding index, Cerebral vasomotor reactivity

\section{Background}

Reversible cerebral vasoconstriction syndrome (RCVS) is characterized by reversible multifocal narrowing of the cerebral arteries with typical manifestations of recurrent thunderclap headache with or without focal neurologic deficits [1]. In patients with RCVS, thunderclap headache is often triggered by the Valsalva maneuver or emotional stress, implicating a dysregulation of cerebral arterial tone with increased sympathetic stimuli.

The mechanism of RCVS is not fully understood. To date, abnormal heart rate variability [2], reduced circulating

\footnotetext{
*Correspondence: cspaul@naver.com

${ }^{\dagger}$ Equal contributors

Samsung Medical Center, Sungkyunkwan University School of Medicine,

Neurology, 81 Irwon-Ro, Gangnam-Gu, Seoul 06531, South Korea
}

endothelial progenitor cells [3], increased oxidative stress [4], and brain-derived neurotrophic factor (BDNF) polymorphism [5] have been reported to play a pathogenic role in RCVS. Recently, reduced cerebral vasomotor reactivity, which represents cerebral endothelial dysfunction, was found in seven of eight selected patients with RCVS in a small retrospective study [6]. However, cerebral endothelial dysfunction is also present in migraineurs, especially in the posterior circulation but also in the anterior circulation in chronic patients $[7,8]$. To identify a disease-specific pattern of cerebral endothelial dysfunction, we aimed to investigate cerebral endothelial function in patients with RCVS compared with age-matched migraineurs and healthy controls.
Springer Open

(c) The Author(s). 2017 Open Access This article is distributed under the terms of the Creative Commons Attribution 4.0 International License (http://creativecommons.org/licenses/by/4.0/), which permits unrestricted use, distribution, and reproduction in any medium, provided you give appropriate credit to the original author(s) and the source, provide a link to the Creative Commons license, and indicate if changes were made. 


\section{Methods}

\section{Participants}

We prospectively recruited patients with RCVS within 1 month of onset at the Samsung Medical Center from April 2015 to July 2016. For the control group, we recruited age-matched patients with episodic migraine without aura (MOA) and healthy controls, with a 1:1:1 patient-to-control ratio from April 2015 to July 2016. This study was approved by the Institutional Review Board in the Samsung Medical Center. All the diagnoses of RCVS and MOA were based on the third edition of the International Classification of Headache Disorders (ICHD-3 beta). Healthy controls did not have any history of migraine, hypertension, diabetes mellitus, hyperlipidemia, stroke, cardiac disease, smoking, or the use of angiotensin II receptor blocker (ARB), angiotensin converting enzyme inhibitor (ACEi) or statin treatments.

\section{Clinical evaluation}

All patients and controls were interviewed by investigators (HAC, MJL, and C-SC), using a structured headache questionnaire on the headache characteristics, medical history, and current medications. For the RCVS group, neurological complications including focal neurological deficits, seizure, ischemic stroke, subarachnoid hemorrhage $(\mathrm{SAH})$, and posterior reversible encephalopathy syndrome (PRES) were evaluated through clinical observation and neuroimaging. All patients with RCVS underwent contrastenhanced brain magnetic resonance imaging (MRI) and magnetic resonance angiogram (MRA). Reversibility was confirmed in all patients with RCVS with a follow-up MRA 3-6 months after symptom onset.

\section{Measurement of cerebral endothelial dysfunction}

To determine cerebral endothelial dysfunction, endotheliumdependent vasodilatory function was measured using transcranial Doppler (TCD) during a breath-holding maneuver. TCD tests were performed within 1 week of diagnosis in the RCVS group, between thunderclap headache attacks. For the MOA group, TCD assessment was performed in the interictal period following $48 \mathrm{~h}$ without headache. The TCD evaluation was performed using a Pioneer TC 8080 (Nicolet Vascular, Madison, WI, USA) by experienced neurophysiologists who were blinded to clinical information.

The 2-MHz Doppler hand-held probe was fixed manually in a supine position. The baseline mean flow velocity (MFV) of bilateral middle cerebral arteries (MCAs), posterior cerebral arteries (PCAs), and the basilar artery (BA) were determined initially. Patients were asked to hold their breath for $30 \mathrm{~s}$ and keep breathing normally until the initiation of breath-holding procedure to avoid the Valsalva manoeuver or hyperventilation. Patients who were unable to hold their breath for the required period were reassessed up to three times. MFV was thoroughly monitored and recorded at the end of the 30-s breathholding maneuver [9-11]. The breath-holding index (BHI) was calculated as the \% increment of the MFV (i.e., [MFV at the end of breath-holding - baseline MFV] $\mathrm{x}$ 100 /baseline MFV) divided by the duration of the breathholding (30 s) [10]. The BHI was determined in bilateral MCAs, PCAs, and the BA. In the RCVS group, the BHI measurement was repeated in selected patients after 3 months.

\section{Statistical analyses}

Statistical analyses were performed using commercially available SPSS version 18.0 software (SPSS Inc., Chicago, IL, USA). Variables were presented as a proportion, mean \pm standard deviation (SD), or a median and interquartile range (IQR). Bivariate analyses were performed using Student's t-tests and Mann-Whitney tests for continuous variables, and Chi-squared and Fisher's exact tests for categorical variables. Independent t-tests were used for the case-control comparison of the MFVs and $\mathrm{BHI}$ in each vessel. To adjust the baseline flow velocities, analysis of covariance (ANCOVA) tests were performed to compare the BHIs in each vessel. The mean BHIs in all tested arteries in each patient was used to group patients into the higher vs lower mean BHI subgroups, using the median value as a cutoff. Demographics, clinical characteristics, and neurologic complications were compared between the two groups via linear regression analysis. Two-tailed $p$-values $<0.05$ were used to establish a statistical significance.

\section{Results}

Baseline demographics of RCVS patients

A total of 34 patients with RCVS were recruited during the study period. Among them, six patients were excluded, since they had received treatment with nimodipine $(n=4)$ or refused to participate in the study $(n=2)$. Thus, 28 patients who completed the TCD assessment per protocol were included. Age-matched migraineurs and healthy controls (mean age, 49.8 years; age range, 30-63 years) were also recruited. The baseline demographics of participants are presented in Table 1. Patients with RCVS had a higher frequency of hyperlipidemia ( $p=0.004$, compared with healthy control), and thus more of these patients were currently being treated with statin medication $(p=0.023$; Table 1$)$ at the time of study.

\section{Transcranial doppler findings in RCVS}

Patients with RCVS underwent TCD tests at a median of 7.5 (IQR, 4.0-16.5) days after onset. There were no significant complications during the breath-holding maneuver. Patients and healthy controls tolerated the breath hold well, except one patient who complained of headache. 
Table 1 Baseline demographics of patients with reversible cerebral vasoconstriction syndrome, migraineurs, and healthy controls

\begin{tabular}{|c|c|c|c|c|c|}
\hline & \multirow[t]{2}{*}{ RCVS $(N=28)$} & \multirow[t]{2}{*}{ Migraine $(N=28)$} & \multirow{2}{*}{$\begin{array}{l}\text { Healthy controls } \\
(N=28)\end{array}$} & \multicolumn{2}{|l|}{$p$-value } \\
\hline & & & & $\overline{\text { vs migraine }^{a}}$ & vs $H^{a}$ \\
\hline Age & $49.8 \pm 10.08(30-63)$ & $49.8 \pm 10.08(30-63)$ & $49.8 \pm 10.08(30-63)$ & $>0.999$ & $>0.999$ \\
\hline Female sex & $24(85.7 \%)$ & $24(85.7 \%)$ & 25 (89.3\%) & $>0.999$ & $>0.999$ \\
\hline Diabetes mellitus & $4(14.3 \%)$ & $0(0 \%)$ & $0(0 \%)$ & 0.111 & 0.111 \\
\hline Hypertension & $4(14.3 \%)$ & $1(3.6 \%)$ & $0(0 \%)$ & 0.611 & 0.236 \\
\hline Hyperlipidemia & $8(28.6 \%)$ & $3(10.7 \%)$ & $0(0 \%)$ & 0.177 & 0.004 \\
\hline Stroke & $0(0 \%)$ & $0(0 \%)$ & $0(0 \%)$ & & \\
\hline Cardiac disease & $1(3.6 \%)$ & $0(0 \%)$ & $0(0 \%)$ & $>0.999$ & $>0.999$ \\
\hline Smoking & $1(3.6 \%)$ & $0(0 \%)$ & $0(0 \%)$ & $>0.999$ & $>0.999$ \\
\hline \multicolumn{6}{|l|}{ Current medication } \\
\hline ARB & $1(3.6 \%)$ & $0(0 \%)$ & $0(0 \%)$ & $>0.999$ & $>0.999$ \\
\hline ACE inhibitor & $1(3.6 \%)$ & $0(0 \%)$ & $0(0 \%)$ & $>0.999$ & $>0.999$ \\
\hline Statin & $6(21.4 \%)$ & $1(3.6 \%)$ & $0(0 \%)$ & 0.101 & 0.023 \\
\hline History of migraine & $6(21.4 \%)$ & $28(100.0 \%)$ & $0(0 \%)$ & $<0.001$ & 0.023 \\
\hline \multicolumn{6}{|l|}{ Causes of RCVS } \\
\hline Idiopathic & $24(85.7 \%)$ & & & & \\
\hline Postpartum & $4(14.3 \%)$ & & & & \\
\hline Medication & $0(0 \%)$ & & & & \\
\hline \multicolumn{6}{|l|}{ Headache } \\
\hline Thunderclap onset & $24(85.7 \%)$ & & & & \\
\hline Triggered by typical precipitants & $17(60.7 \%)$ & & & & \\
\hline Recurrent during first 1 month & 19 (67.9\%) & & & & \\
\hline \multicolumn{6}{|l|}{ Neurological complication } \\
\hline Neurological deficits & $1(3.6 \%)$ & & & & \\
\hline Seizure & $3(10.7 \%)$ & & & & \\
\hline Cerebral infarction & $0(0 \%)$ & & & & \\
\hline Cortical SAH & $1(3.6 \%)$ & & & & \\
\hline PRES & $2(7.1 \%)$ & & & & \\
\hline
\end{tabular}

$A C E$ angiotensin converting enzyme, $A R B$ angiotensin II receptor blocker, PRES posterior reversible encephalopathy syndrome, $R C V S$ reversible cerebral vasoconstriction syndrome, $S A H$ subarachnoid hemorrhage

Values are presented as number (\%), mean \pm standard deviation (ranges). ${ }^{\text {acompared }}$ with RCVS

Baseline mean flow velocities were higher in the RCVS group in bilateral MCAs and the BA, compared with migraineurs $(p=0.059$ for the left MCA, $p=0.046$ for the right $\mathrm{MCA}$, and $p=0.016$ for the $\mathrm{BA}$; Table 2) and healthy controls $(p=0.032$ for the left MCA, $p=0.041$ for the right MCA, and $p=0.034$ for the BA; Table 2). Nine of twenty-eight patients with RCVS (32.1\%) presented increased MFVs in the MCAs that exceeded the upper limit of the normal range $(80 \mathrm{~cm} / \mathrm{s})$. Among them, four patients $(14.3 \%)$ met the criteria for mild vasospasm $(>120 \mathrm{~cm} / \mathrm{s})$. There were no significant differences in the flow velocities of bilateral PCAs.

All patients of RCVS showed significantly lower BHIs in basal arteries compared with healthy controls. In addition, patients with RCVS showed lower BHIs in the anterior circulation compared to subjects with episodic MOA (Table 2). Differences in the BHIs between the RCVS and MOA groups were not significant in the posterior circulation, and the results were generally unchanged after controlling for baseline MFVs. Similar results were observed even when the six migraineurs in the RCVS group were excluded (Additional file 1: Table S1-S2).

\section{Factors related to cerebral endothelial dysfunction in RCVS}

When patients with RCVS were stratified according to a higher or lower BHIs, vascular risk factors were not associated with endothelial dysfunction (Table 3). Patients with lower BHIs were younger and had more migraines than those with higher BHIs, although statistical significance between groups was not reached. 
Table 2 Cerebral blood flow velocities and breath-holding indices

\begin{tabular}{|c|c|c|c|c|c|}
\hline & \multirow[t]{2}{*}{ RCVS $(N=28)$} & \multirow[t]{2}{*}{ Migraine $(N=28)$} & \multirow[t]{2}{*}{ Healthy controls $(N=28)$} & \multicolumn{2}{|c|}{$p$-value (adjusted $p$ ) } \\
\hline & & & & vs migraine $^{a}$ & vs $\mathrm{HC}^{\mathrm{a}}$ \\
\hline \multicolumn{6}{|c|}{ Mean flow velocities $(\mathrm{cm} / \mathrm{s})$} \\
\hline L MCA & $76.4 \pm 28.56$ & $64.2 \pm 11.20$ & $62.5 \pm 10.47$ & 0.059 & 0.032 \\
\hline R MCA & $78.4 \pm 28.05$ & $65.9 \pm 14.04$ & $66.1 \pm 10.32$ & 0.046 & 0.041 \\
\hline LPCA & $38.6 \pm 10.75$ & $36.5 \pm 6.65$ & $37.4 \pm 6.58$ & 0.410 & 0.617 \\
\hline R PCA & $39.4 \pm 11.29$ & $36.8 \pm 6.36$ & $38.8 \pm 7.14$ & 0.341 & 0.906 \\
\hline BA & $54 \pm 13.61$ & $46.7 \pm 5.98$ & $46.5 \pm 10.65$ & 0.016 & 0.034 \\
\hline \multicolumn{6}{|c|}{ Breath-holding index } \\
\hline L MCA & $0.8 \pm 0.33$ & $1.1 \pm 0.34$ & $1.2 \pm 0.24$ & $0.002(0.004)$ & $<0.001(<0.001)$ \\
\hline R MCA & $1.0 \pm 0.36$ & $1.3 \pm 0.52$ & $1.3 \pm 0.39$ & $\mathbf{0 . 0 3 8}(0.109)$ & $0.009(0.033)$ \\
\hline LPCA & $0.8 \pm 0.42$ & $1.0 \pm 0.38$ & $1.3 \pm 0.43$ & $0.069(0.160)$ & $<0.001(<0.001)$ \\
\hline R PCA & $1.0 \pm 0.42$ & $1.1 \pm 0.34$ & $1.3 \pm 0.49$ & $0.247(0.375)$ & $\mathbf{0 . 0 2 8}(0.090)$ \\
\hline $\mathrm{BA}$ & $0.9 \pm 0.47$ & $1.0 \pm 0.42$ & $1.1 \pm 0.31$ & $0.120(0.178)$ & $0.060(\mathbf{0 . 0 3 1 )}$ \\
\hline
\end{tabular}

$B A$ basilar artery, $\mathrm{HC}$, healthy control, $M C A$ middle cerebral artery, $P C A$ posterior cerebral artery, $R C V S$ reversible cerebral vasoconstriction syndrome Values are presented as mean \pm standard deviation. ${ }^{a}$ compared with RCVS

Adjustment of flow velocities were performed to compare breath holding indices in each vessel

\section{Follow-up evaluation in RCVS}

Ten RCVS patients underwent follow-up BHI studies after 3 months. Follow-up study results showed complete normalization in seven patients (pre/post, $0.8 \pm 0.22 / 1.3 \pm 0.46$ ), while three patients did not show a significant improvement (no change, $n=2$; aggravation, $n=1$ ) (Fig. 1).

Table 3 Factors related to cerebral endothelial dysfunction in RCVS

\begin{tabular}{llll}
\hline & Low BHI $(\mathrm{N}=14)$ & High BHI (N = 14) & P-value \\
\hline Age & $46.9 \pm 10.93(30-60)$ & $52.7 \pm 8.55(35-63)$ & 0.126 \\
Female sex & $13(92.9 \%)$ & $11(78.6 \%)$ & 0.297 \\
Diabetes mellitus & $2(14.3 \%)$ & $2(14.3 \%)$ & $>0.999$ \\
Hypertension & $1(7.1 \%)$ & $2(14.3 \%)$ & 0.558 \\
Hyperlipidemia & $5(35.7 \%)$ & $3(21.4 \%)$ & 0.422 \\
Stroke & $0(0 \%)$ & $0(0 \%)$ & \\
Cardiac disease & $1(7.1 \%)$ & $0(0 \%)$ & 0.327 \\
Smoking & $0(0 \%)$ & $1(7.1 \%)$ & 0.327 \\
Current medication & & & 0.276 \\
$\quad$ ARB & $0(0 \%)$ & $1(7.1 \%)$ & 0.385 \\
ACE inhibitor & $1(7.1 \%)$ & $0(0 \%)$ & 0.376 \\
\multicolumn{1}{c}{ Statin } & $2(14.3 \%)$ & $4(28.6 \%)$ & 0.069 \\
History of migraine & $5(35.7 \%)$ & $1(7.1 \%)$ & 0.297 \\
Causes of RCVS & & & \\
Idiopathic & $11(78.6 \%)$ & $13(92.9 \%)$ & \\
Postpartum & $3(21.4 \%)$ & $1(7.1 \%)$ & $(0 \%)$ \\
Medication & $0(0 \%)$ & & \\
\hline ACE angotensin & & & \\
\hline
\end{tabular}

$A C E$ angiotensin converting enzyme, $A R B$ angiotensin II receptor blocker, $R C V S$ reversible cerebral vasoconstriction syndrome

Values are presented as number (\%), mean \pm standard deviation (ranges)

\section{Discussion}

The key findings of this article are as follows: (1) cerebral endothelial dysfunction is present in patients with RCVS, (2) the pattern of endothelial dysfunction is more widespread in RCVS than in migraine, and (3) endothelial dysfunction is reversible in most, but not all patients. Impaired cerebral endothelial dysfunction may play a

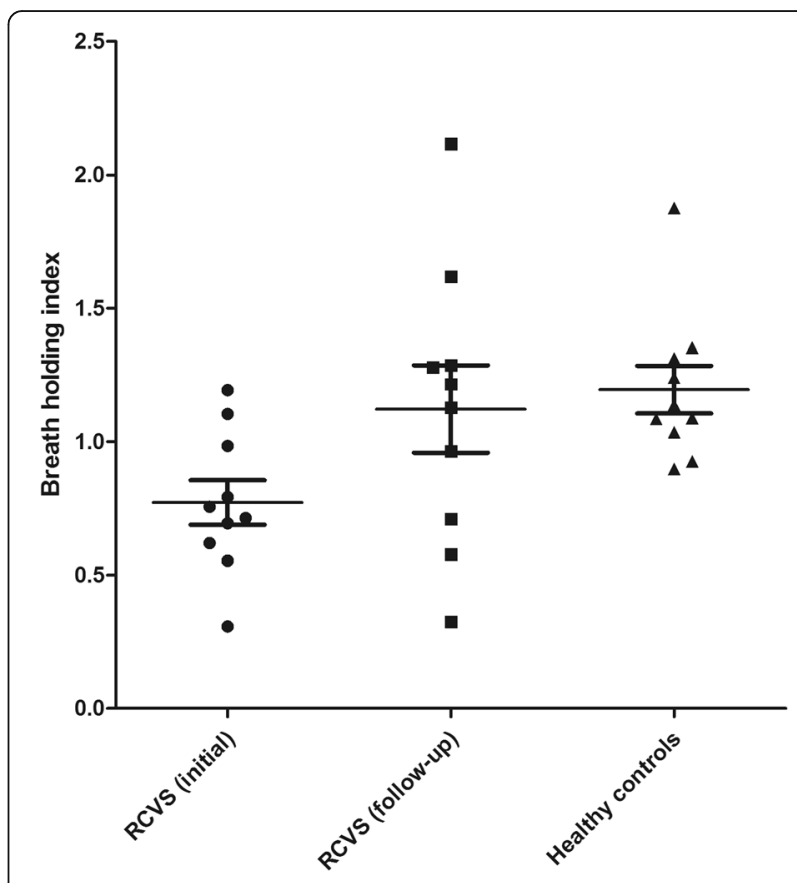

Fig. 1 Baseline and follow-up breath holding indices in patients and matched controls Breath holding index (BHI) of all tested vessels was averaged. The follow-up study showed a complete normalization in seven patients, no change in two, and aggravation in one patient 
role in the pathophysiology of RCVS; its role in predisposition should be determined in future researches.

The endothelium, which comprises the innermost layer of the vessel wall, is a key mediator of vascular tone since it controls vasoconstriction and vasodilation [12]. Endothelial cell function can be assessed in two manners. One method of assessment is measuring circulating markers of endothelial cell activation, injury, or repair [12, 13]. In a recent study, Chen et al. [3] reported a reduced number of circulating markers that is responsible for endothelial cell repair in patients with RCVS. The other method of measuring endothelial cell function is the assessment of endothelium-dependent vasomotion [12]. Unlike circulating markers, endothelium-dependent vasomotion can be measured differently in systemic and cerebral circulation. Cerebral vasomotor reactivity is a method that specifically measures endothelium-dependent vasodilation in cerebral circulation, which may differ from systemic endothelial function [14, 15]. Our findings confirm that endothelium-dependent vasodilation is impaired in the cerebral arteries of patients with RCVS. Along with the findings of cardiac involvement in RCVS [15], future research should focus on the correlation or difference between cerebral and systemic endothelial dysfunction in RCVS.

Breath holding is a physiologic and reliable method to induce endothelium-dependent vasodilation in cerebral arteries in response to arterial carbon dioxide levels $[10,16]$. The BHI is calculated using the \% increment of postbreath-holding MFVs compared to baseline MFVs. In the present study, patients with RCVS showed significantly reduced BHIs in all basal arteries, implying clinical significance of impaired endothelium-dependent vasodilation in RCVS. There was, however, not a great difference in the posterior circulation in RCVS compared with episodic MOA. Cerebral endothelial dysfunction in the posterior circulation has been consistently reported in migraine [8]. Two hypotheses can explain the differences in the distribution of cerebral endothelial dysfunction between RCVS and migraine. First, endothelial function may be systemically impaired in patients with RCVS to a greater extent than that in migraineurs $[3,14,15]$. Second, as the posterior circulation is less innervated with sympathetic fibers than the anterior circulation [17], both sympathetic and parasympathetic dysfunction may play a role in the pathophysiology of RCVS [2].

Our study showed that endothelial dysfunction in RCVS is not related to advanced age, vascular risk factors, or premorbid medications. Rather, younger age and premorbid migraine were associated with more severe endothelial dysfunction, although statististically insignificant. However, it is still not clear if cerebral endothelial dysfunction is a predisposition of RCVS or a part of its pathophysiology. Although our study was not longitudinally designed, our limited data showed a normalization of endothelial dysfunction in some patients. However, endothelial dysfunction was persistent or even aggravated in a small number of patients, suggesting either poor treatment outcome or premorbid endothelial dysfunction. Future studies should be designed to determine whether endothelial dysfunction is a predisposition for RCVS or not. In addition, other predisposing factors such as genetic polymorphism should be considered in association with endothelial dysfunction [5].

This study has strengths. First, this is a prospective case-control study that included RCVS, migraine, and healthy control subjects. Confounding factors that might affect cerebral microvasculature were thoroughly investigated and controlled for in the analysis. Second, we selectively measured cerebral-specific endothelial function in RCVS. Third, we made several hypotheses regarding the pathogenesis of RCVS. However, this study has some limitations. First, this study was performed in a single ethnic population, i.e. Asians. Our study subjects had a small number of neurological complications, which might be attributed to the study population. External validation is warranted in different populations. Second, we did not evaluate concurrent systemic endothelial dysfunction in comparison to the BHI. It would be worthwhile to test the correlation or difference between systemic endothelial dysfunction (i.e. flow-mediated dilatation) and the BHI in future studies.

\section{Conclusions}

In conclusion, our results suggest that cerebral endothelial function is impaired in patients with RCVS in a widespread pattern. Future investigations should be conducted to reveal differences in systemic and cerebral endothelial function and the implications of endothelial dysfunction in the pathogenesis and neurological outcomes of patients with RCVS.

\section{Additional file}

Additional file 1: Table S1. Baseline demographics of patients with reversible cerebral vasoconstriction syndrome, migraineurs, and healthy controls. Table S2. Cerebral blood flow velocities and breath-holding indices. (DOC $66 \mathrm{~kb}$ )

\section{Abbreviations}

ACEi: Angiotensin converting enzyme inhibitor; ARB: Angiotensin II receptor blocker; BA: Basilar artery; BDNF: Brain-derived neurotrophic factor;

BHI: Breath-holding index; ICHD-3 beta: International Classification of Headache Disorders; IQR: Interquartile ranges; MCAs: Middle cerebral arteries; MFV: Mean flow velocity; MOA: Migraine without aura; MRA: Magnetic resonance angiogram; MRI: Magnetic resonance imaging; PCAs: Posterior cerebral arteries; PRES: Posterior reversible encephalopathy syndrome; RCVS: Reversible cerebral vasoconstriction syndrome; SAH: Subarachnoid hemorrhage; SD: Standard deviation; TCD: Transcranial doppler 


\section{Acknowledgment}

The authors thank Ms. Miran Jung for her help with data management.

\section{Funding}

This study was supported by a grant of the Korean Health Technology R\&D

Project, Ministry of Health \& Welfare, Republic of Korea (HI13C1521).

\section{Authors' contributions}

HAC conceived and designed the study, acquired, analyzed and interpreted data, drafted and revised the manuscript. MJL conceived and designed the study, acquired, analyzed and interpreted data, drafted and revised the manuscript. CSC conceived and designed the study, and revised the manuscript for intellectual content. All authors read and approved the final manuscript.

\section{Competing interests}

The authors declare that they have no competing interests.

Received: 13 December 2016 Accepted: 15 February 2017

Published online: 23 February 2017

\section{References}

1. The International Classification of Headache Disorders (2013) 3rd edition (beta version). Cephalalgia 33:629-808

2. Chen SP, Yang AC, Fuh JL, Wang SJ (2013) Autonomic dysfunction in reversible cerebral vasoconstriction syndromes. J Headache Pain 14:94

3. Chen SP, Wang YF, Huang PH, Chi CW, Fuh JL, Wang SJ (2014) Reduced circulating endothelial progenitor cells in reversible cerebral vasoconstriction syndrome. J Headache Pain 15:82

4. Chen SP, Chung YT, Liu TY, Wang YF, Fuh JL, Wang SJ (2013) Oxidative stress and increased formation of vasoconstricting F2-isoprostanes in patients with reversible cerebral vasoconstriction syndrome. Free Radic Biol Med 61:243-8

5. Chen SP, Fuh JL, Wang SJ, Tsai SJ, Hong CJ, Yang AC (2011) Brain-derived neurotrophic factor gene Val66Met polymorphism modulates reversible cerebral vasoconstriction syndromes. PLoS One 6:e18024

6. Topcuoglu MA, Chan ST, Silva GS, Smith EE, Kwong KK, Singhal AB. Cerebral vasomotor reactivity in reversible cerebral vasoconstriction syndrome. Cephalalgia. 2016. [Epub ahead of print]

7. Rajan R, Khurana D, Lal V (2015) Interictal cerebral and systemic endothelial dysfunction in patients with migraine: a case-control study. J Neurol Neurosurg Psychiatry 86:1253-7

8. Gonzalez-Quintanilla V, Toriello M, Palacio E, Gonzalez-Gay MA, Castillo J, Montes S et al (2016) Systemic and cerebral endothelial dysfunction in chronic migraine. A case-control study with an active comparator. Cephalalgia 36:552-60

9. Settakis G, Lengyel A, Molnar C, Bereczki D, Csiba L, Fulesdi B (2002) Transcranial Doppler study of the cerebral hemodynamic changes during breath-holding and hyperventilation tests. J Neuroimaging 12:252-8

10. Markus HS, Harrison MJ (1992) Estimation of cerebrovascular reactivity using transcranial Doppler, including the use of breath-holding as the vasodilatory stimulus. Stroke 23:668-73

11. Sharma VK, Tsivgoulis G, Ning C, Teoh HL, Bairaktaris C, Chong VF et al (2008) Role of Multimodal Evaluation of Cerebral Hemodynamics in Selecting Patients with Symptomatic Carotid or Middle Cerebral Artery Steno-occlusive Disease for Revascularization. J Vasc Interv Neurol 1:96-101

12. Verma S, Buchanan MR, Anderson TJ (2003) Endothelial function testing as a biomarker of vascular disease. Circulation 108:2054-9

13. Deanfield JE, Halcox JP, Rabelink TJ (2007) Endothelial function and dysfunction: testing and clinical relevance. Circulation 115:1285-95

14. Perko D, Pretnar-Oblak J, Sabovic M, Zaletel M, Zvan B (2011) Associations between cerebral and systemic endothelial function in migraine patients: a post-hoc study. BMC Neurol 11:146

15. John S, Hajj-Ali RA, Min D, Calabrese LH, Cerejo R, Uchino K (2015) Reversible cerebral vasoconstriction syndrome: Is it more than just cerebral vasoconstriction? Cephalalgia 35:631-4

16. Tancredi FB, Hoge RD (2013) Comparison of cerebral vascular reactivity measures obtained using breath-holding and $\mathrm{CO} 2$ inhalation. J Cereb Blood Flow Metab 33:1066-74

17. Gulbenkian S, Uddman R, Edvinsson L (2001) Neuronal messengers in the human cerebral circulation. Peptides 22:995-1007

\section{Submit your manuscript to a SpringerOpen ${ }^{\circ}$ journal and benefit from:}

- Convenient online submission

- Rigorous peer review

- Immediate publication on acceptance

- Open access: articles freely available online

- High visibility within the field

- Retaining the copyright to your article

Submit your next manuscript at $>$ springeropen.com 